Research Article

\title{
Prediction-Based Maintenance of Existing Bridges Using Neural Network and Sensitivity Analysis
}

\author{
Pengyong Miao \\ Graduate School of Engineering, Hokkaido University, Sapporo, Japan \\ Correspondence should be addressed to Pengyong Miao; miaopengyong@eis.hokudai.ac.jp
}

Received 9 June 2021; Accepted 15 July 2021; Published 28 July 2021

Academic Editor: Pengfei Liu

Copyright (C) 2021 Pengyong Miao. This is an open access article distributed under the Creative Commons Attribution License, which permits unrestricted use, distribution, and reproduction in any medium, provided the original work is properly cited.

\begin{abstract}
Bridge deterioration is affected by various factors. However, neither the relationships between these factors and deterioration are explicitly determined, nor the relative effect of each factor on deterioration is well understood. This study proposed a methodology to resolve these issues by integrating an artificial neural network (ANN) and sensitivity analysis method. The ANN was used to predict deterioration, and the sensitivity analysis method was applied to evaluate the influence of each factor on deterioration. Testing the methodology with 3,368 bridge inspection data pieces indicates that (1) the developed ANN obtained an accuracy of about 65\%; and (2) seven factors were identified affecting deterioration. The established ANN model has equivalent performance for three deterioration grades and four types of bridges. Two sensitivity analysis (the Shapley value and the Sobol indices) methods were compared, and they identified the same five most important factors. Consequently, the methodology can effectively avoid the uncertainty of factors on deterioration by providing a relative importance list of factors. The methodology's predictive ability and factor importance identification ability make it suitable for decision-makers to understand the deterioration situations and to schedule a further inspection and corresponding maintenance strategies.
\end{abstract}

\section{Introduction}

Bridges that are in use for many years suffer various degrees of deterioration. Many studies have been conducted to evaluate the impacts of individual factors on deterioration [1]. However, the practical bridge is a complicated system project, the relationships between deterioration-related factors and deterioration are not explicitly determined, neither is the relative impact of each factor on deterioration. Maintenance engineers inspect and evaluate the conditions of bridges and record these results at regular intervals. Over time, databases with large numbers of maintenance items are obtained, which provides a possibility to establish the relationships between factors and deterioration and to assess the impact of each factor. In addition, proper predictionbased maintenance strategies are more economical to keep the soundness of bridges than corrective maintenance, because the corrective maintenance focuses on postfailure repair and is usually insufficient to ensure structural safety [2]. Furthermore, the inspection is labor-intensive and time- consuming. Therefore, there are untapped potentials and needs to utilize maintenance databases for predicting deterioration to determine seriously deteriorated bridge for implementing further inspection and making maintenance strategies. To fulfill these needs, an inspection database is brought into new data processing methods (including neural network and sensitivity analysis) to predict deterioration in grade and to determine the factors affecting deterioration. The potentially influencing factors include bridge design and construction quality, bridge geometry, materials, repairs, traffic volume, and surrounding environments.

Appropriate artificial neural network (ANN) architectures can be trained on the maintenance databases to predict the future deterioration situations [3]. The noteworthy characteristic of an ANN is its learning ability. In the process of establishing an ANN model, the network processes the inputs and obtains an output, compares the output with the ideal response, and calculates the errors. Then the parameters of connections ( $W_{1}$ and $W_{2}$ in Figure 1) are updated according to the errors. If the network paradigm and 


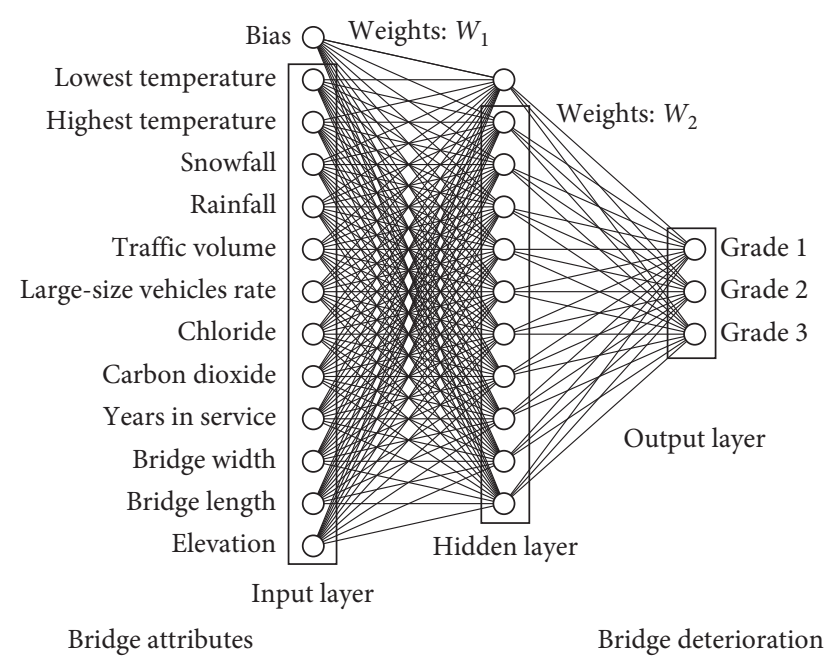

Figure 1: Configuration of a neural network used for prediction.

parameters are appropriately designed, these can result in satisfactory forecasting performance. In the maintenance of existing bridges, an ANN can assist maintenance work in establishing the relationships between the potential factors and the deterioration grades of bridges to predict the deterioration.

Since an ANN is usually trained by using a set of data from a database, the results only explain the relationships between all inputs (factors) and output (prediction). Through the relationships, it is difficult to understand the contribution of each factor on a specific prediction. Therefore, the relationship established by an ANN becomes a "black box" [4]. Although an ANN model can provide prediction and show better performance, its application in the practical maintenance of bridges is limited owing to the ignorance of the influence of each factor on the prediction.

In summary, not only should the prediction model using an ANN be established to determine the deterioration situations but also each factor's importance should be calculated to understand the reasons for deterioration. In addition, the applied database must contain high-quality data for formulating a deterioration prediction model with optimal performance and making convincing explanations for factors affecting deterioration. Therefore, the data quality of the applied inspection database must be checked carefully before it is conducted in analysis.

This study introduced the development and verification of a proposed methodology that can be used to predict bridge deterioration and to determine the reasons for deterioration. Specifically, an ANN was applied to analyze an inspection database for establishing a prediction model, whereby the deterioration grades can be predicted to determine the seriously deteriorated bridge. The performance of the established ANN model was evaluated for different deterioration grades and different types of bridges. In addition, the Shapley value method $[5,6]$ and the Sobol indices $[6,7]$ were used to calculate the importance of all factors. Furthermore, the Shapley value method were applied to the prediction model for further analyzing the estimated importance, broken down by factors, deterioration grades, bridge types, and environments. Finally, two bridges were taken as examples to illustrate the practical application of the proposed method.

\section{Background}

Maintenance is essential to keep structures sound, serviceable, and safe. In many countries, condition assessment of existing bridges is carried out mainly through periodic inspections [8-10]. Based on the assessment results, necessary interventions or further inspections are scheduled. Since prediction-based maintenance can prevent further deterioration and keep bridges in sound conditions with lower costs compared with corrective maintenance, prediction-based maintenance is widely used [2]. Predictionbased maintenance depends heavily on the accurate prediction of deterioration. Potential approaches are described below.

The approaches commonly used in predicting deterioration include two categories: the deterministic model and the probabilistic model [11]. The deterministic model deals with certain and known variables. Hyman et al. [12] pointed out that deterioration is related to the age of bridge through a piecewise linear regression model. Busa et al. [13] used linear regression to model bridge deterioration and concluded that age and daily traffic are two important factors. Abu-Tair [14] discussed the application of the factor method in predicting deterioration and noted that this method is highly subjective. Pan et al. [15] presented a matrix-driven fuzzy linear regression model to predict bridge conditions. Similarly, Kim et al. [16] applied a sigmoid function and nondestructive evaluation data to evaluate the deterioration of a bridge deck. Jeong et al. [17] developed a nonlinear regression model for determining the expected service life of a bridge. Although the performance of bridges in their service life can be described by the formulas of deterministic models, these models ignored the uncertainty of deterioration [18].

In addition, some researchers have focused on probabilistic models, in which the mostly used deterioration model is the Markov chain. Li et al. [19] and Wellalage et al. [20] used the Markov chain-based deterioration model to predict bridge conditions. However, an optimal transition probability matrix is difficult to obtain. Agrawal et al. [18] compared the Markov chain with the Weibull distribution and concluded the later method would be better. Time-based models present the time distribution of the duration taken by bridges to change their condition from one to another. A set of variables (factors) are used for this process such as environmental exposure, design attributes, and maintenance schedule. These types of models were used in the studies done by Mauch and Madanat [21] and Lee et al. [22]. In their approaches, a series of random variables (factors) with different distributions determined the performance of bridges.

Except for the deterministic and probabilistic models, the ANN has been applied to practical maintenance engineering [23-25], as it can solve multivariate problems. In the application of the ANN to maintenance, Sobanjo [26] used 
the inspection records for 50 bridge superstructures to predict bridge deterioration that inputs only the age of bridge and outputs the deterioration condition of bridge superstructure. Huang [27] identified eleven significant factors and developed an ANN model with these factors to predict deterioration of bridge decks. Using the same operation, Al-Hussein [11] designed an ANN to estimate the deterioration by considering the structure types, component types, exposure environments, and defects. Lim and Chi [28] considered the factors of identification, structure, inspection, and environment, and developed an ANN to predict the number of damage occurrences on bridge decks and their severity with the accuracy of about $95 \%$. None of these researchers discussed, however, the specific influences of factors on bridge deterioration.

Inspired by these studies, we applied the ANN to analyze an inspection database towards predicting deterioration of existing bridges to optimize further inspection and formulate maintenance strategies. However, Zhang et al. [4] and Caruana et al. [29] noted that the ANN can give rise to a black box problem. Although the ANN model can predict deterioration given influencing factors, the prediction model provides only one predicted value without knowing the reasons for deterioration. This makes it difficult for engineers to trust the prediction, to understand what factors affect the prediction, and to determine targeted intervention. To solve these difficulties, the proposed methodology integrates an ANN and the sensitivity analysis method. An ANN predicts the deterioration in grades, and the sensitivity analysis method calculates the relative importance of factors on deterioration.

\section{Proposed Methodology}

The methodology consists of the following two parts: a prediction model and sensitivity analysis approaches, as shown in Figure 2.

3.1. Prediction Part. Accurate prediction of bridge deterioration is essential for the planning of further inspection, maintenance, and repair at lower cost. To predict deterioration, the ANN is applied to analyze an inspection database for establishing a prediction model. Maintenance data with high quality should be extracted and classified into the following two categories: potential factors affecting deterioration and the deterioration grades. Since many factors will affect the deterioration, all potential external and internal factors likely to influence the bridges should be highlighted [30]. The influential factors for consideration include those of bridge design and geometries, applied materials, environment, loading, and others, which will be elaborated in Section 4.1.1. However, this does not mean that each factor has the same impact on the bridge deterioration. Therefore, it is necessary for the proposed methodology to identify the significant factors from the many insignificant factors [31].

Given the characteristics of the ANN, the prediction part can be applied to establish the optimal relationships between the potential factors and the deterioration grades, as

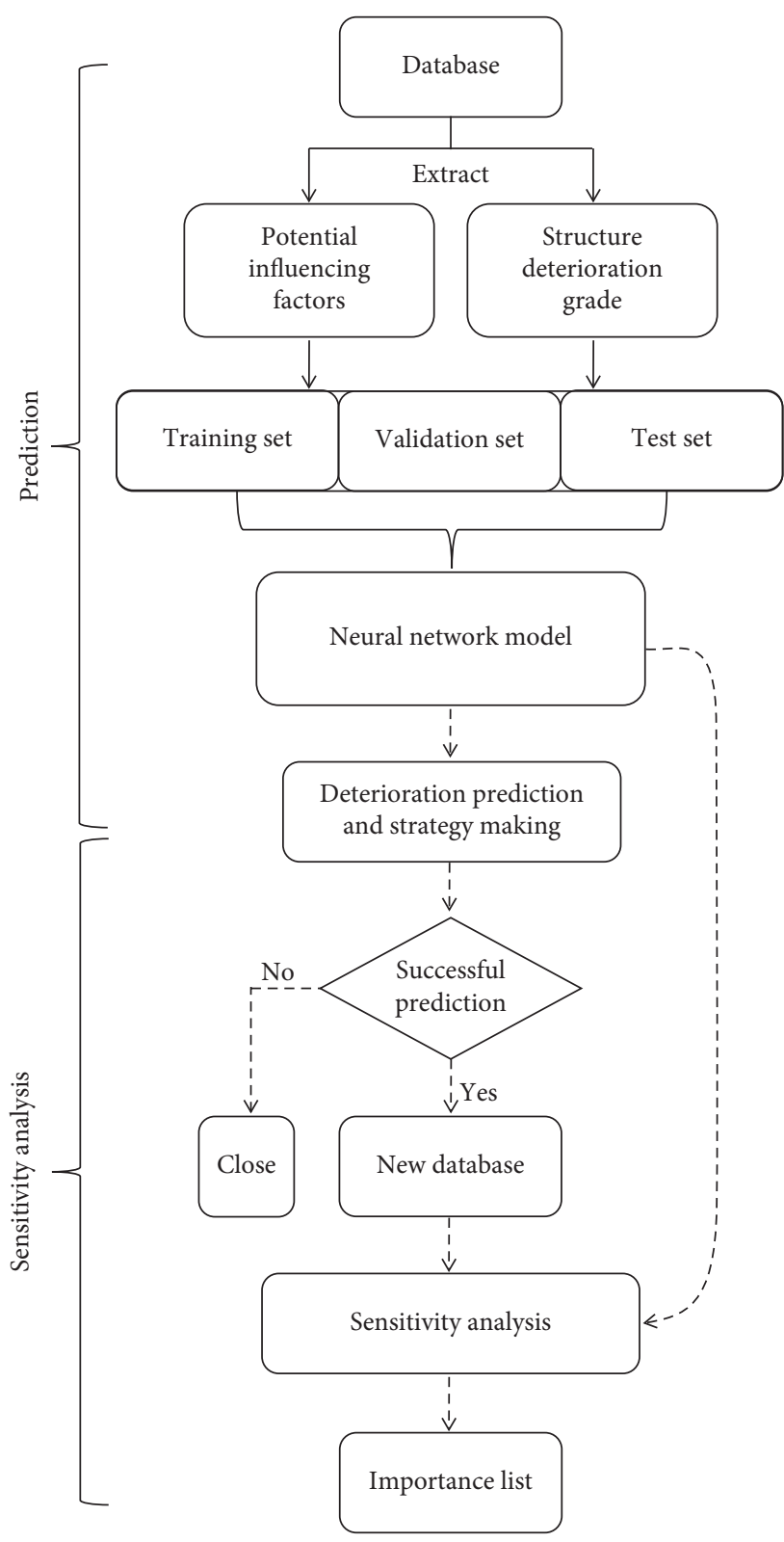

Figure 2: Proposed methodology.

illustrated in Figure 1. Details of ANN refer to Goodfellow et al. [32] and Guliyev [33]. The performance of the prediction model can be improved by continuously integrating new inspection data to update the model. Accordingly, two parameter matrices of $W_{1}$ and $W_{2}$ in Figure 1 can be obtained, which represent the whole relationships between the inputs and the outputs. However, those two parameter matrices make the relationships hard to be understood because they indicate the complicated nonlinear computation. In other words, the contribution of each factoron deterioration is difficult to determine directly from the prediction model.

3.2. Sensitivity Analysis. Sensitivity analysis can be applied to overcome the uncertainty of factors on deterioration, 
because sensitivity analysis can tell engineers what factors affect the deterioration and their relative importance. This study applied two sensitivity analysis methods: Shapley value and Sobol indices, as described below. A prediction can be explained by assuming that each factor of the instance is a "player" in a game and the prediction is the payout. Shapley values show how to fairly distribute the "payout" among the factors [34]. Next, the Shapley value method is briefly introduced. The set of $m$ potentially influencing factors is represented by $N=\left\{x_{1}, x_{2}, \ldots, x_{m}\right\}$, and any permutation $S$ of $N$ is a coalition. The characteristic function $v$ maps all coalitions to "payout," which in our case is the deterioration grade. The function $v$ has the following definitions: if $S$ is a permutation (coalition) of all factors; then $v(S)$ describes the total impact of all factors in coalition $S$ [35]. The value of $v(S)$ is determined by inputting $S$ in the prediction model. follows:

The importance $\varphi_{i}(v)$ of factor $x_{i}$ is determined as

$$
\varphi_{i}(v)=\frac{\sum_{S \subseteq N}(((m-|S|-1) !|S| !) / m !)\left(v\left(S \cup\left\{x_{i}\right\}\right)-v(S)\right)}{\{i\}},
$$

where $m$ is the number of factors. The sum extends over all subsets $S$ of $N$ excluding factor $x_{i}$. The formula can be interpreted as follows: the contribution of factor $x_{i}$ in $S$ is $v\left(S \cup\left\{x_{i}\right\}\right)-v(S)$; the average of this contribution over the possible coalitions is the final contribution of this factor. In our case, $\varphi(v)$ refers to the deterioration grade and is defined as

$$
\varphi(v)=\sum_{i} \varphi_{i}(v)
$$

The Shapley value method can calculate the relative importance of each factor, regardless of the complexity of the prediction model. Therefore, it can explain any prediction model [35]. The calculated importance is related to the magnitude of difference in the prediction values when a factor is considered (such as bridge width) versus when it is not considered (such as when bridge width is unknown) [5]. In summary, the Shapley value method identifies all factors and evaluates their relative importance, enabling an engineer to figure out factors that significantly influence the deterioration and formulate a corresponding intervention strategy.

Except for the Shapley value method, Sobol indices $[6,7]$ is another useful method to detect the sensitivity of predictions to inputs. In this study, these two methods will be compared using an actual case in Section 4.3. Conforming to the descriptions of these two methods, we present the specific steps to achieve the sensitivity analysis of the predictions, as shown in Figure 2. Given values of factors, the deterioration grades can be predicted (Section 3.1), with some predictions being successful and the rest being unsuccessful. All successfully predicted bridges are extracted to form a new database. Since unsuccessful predictions may lead to incorrect importance estimates, the Shapley value and Sobol indices methods are applied to the new database to detect the sensitivity of the prediction model to each factor.

\section{Case Study}

An inspection database of 3,386 bridges in Hokkaido in Japan was employed to verify the effectiveness of the methodology. These bridges are inspected every five years [8] and their locations are shown in Figure 3. The entire verification process will be described from data preparation, establishment of prediction model, and estimation of factor importance.

4.1. Data Preparation. We eliminated incomplete and unreasonable data from the original database and selected 3,368 out of 3,386 bridges. Specifically, the bridges with unknown ages were discarded. In conjunction with the age constrain, the constraint of nondecreasing deterioration grade was introduced. For example, a bridge is assessed grade 2 in an inspection and assessed grade 1 in the next inspection; this kind of bridge was not considered. Then, the data for the selected bridges were divided into potentially influencing factors and the deterioration grades.

4.1.1. Potentially Influencing Factors. Factors that have the potential to affect deterioration were regarded as "inputs" in the prediction model. The bridge features, such as bridge length, bridge width, elevation, and years in service, were extracted from the inspection database. Temperature and carbon dioxide concentration were obtained from Japan Meteorological Agency [36]. Traffic volume was obtained from the traffic survey $[37,38]$. Airborne salt was calculated according to previous studies $[39,40]$. In summary, the potentially influencing factors include bridge geometry, environment, loading conditions, and others, as listed in Table 1 . There are many other factors that would affect the deterioration but are not considered, such as fatigue crack, design, and construction quality. The main reason is the inability to collect relevant data. However, this deficiency will force us to collect more data to improve our future work and encourage modifying the data capturing method in the future inspection. More details are described below for the factors of traffic volume and airborne salt. This study considered four types of bridges, including PC, RC, PC and $\mathrm{RC}$ hybrid, and steel bridges.

(1) Rate of the large-size vehicles

According to the survey of daily traffic volume $[37,38]$, the proportion of large-size vehicles (including bus, lorry, construction heavy equipment, and other special equipment) $R_{v}$ can be calculated by

$R_{v}=\frac{\text { number of large size vehicles daily }}{\text { daily traffic volume }} \times 100 \%$.

(2) Airborne salt

The bridges within $1 \mathrm{~km}$ of the coastline are considered influenced by the airborne salt. The airborne salt concentration can be calculated by the following equation [39]: 


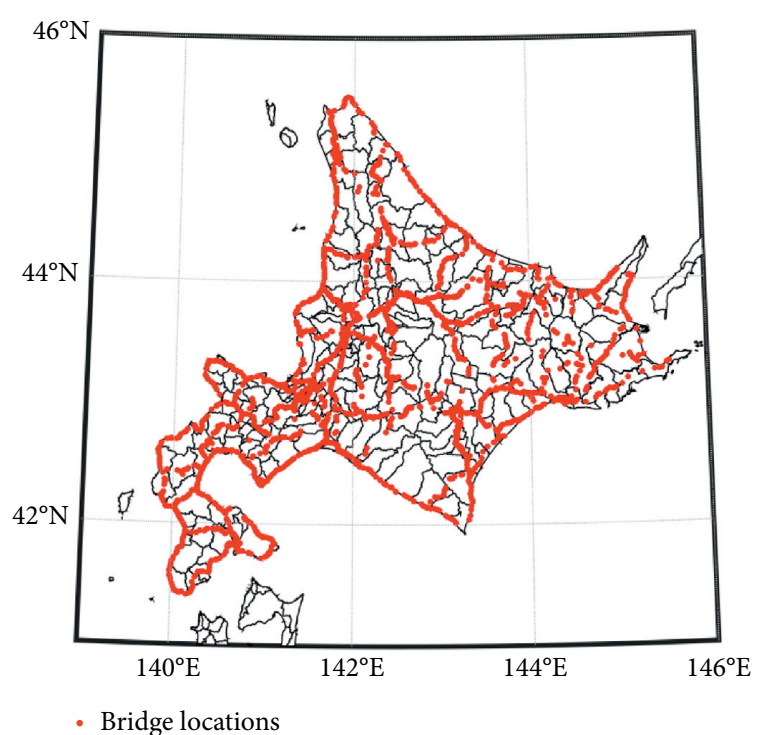

FIGURE 3: Locations of the bridges.

TABLE 1: Potentially influencing factors.

\begin{tabular}{lc}
\hline $\begin{array}{l}\text { Bridge geometry } \\
\text { factors }\end{array}$ & $\begin{array}{c}\text { Attributes (unit) } \\
\text { Width }(\mathrm{m})\end{array}$ \\
\hline Environmental factors & $\begin{array}{c}\text { Elevation }(\mathrm{m}) \\
\text { Yearly highest and lowest temperatures } \\
\left({ }^{\circ} \mathrm{C}\right)\end{array}$ \\
& $\begin{array}{c}\text { Carbon dioxide concentration }(\mathrm{ppm}) \\
\text { Airborne salt concentration }(\mathrm{mdd} \cdot \mathrm{NaCl}) \\
\text { Yearly average snowfall }(\mathrm{cm}) \\
\text { Yearly average rainfall }(\mathrm{cm})\end{array}$ \\
\hline Loading condition & $\begin{array}{c}\text { Daily traffic volume (vehicles/day) } \\
\text { Rate of the large-size vehicles }(\% / \text { day) }\end{array}$ \\
\hline Other & Years in service (years) \\
Bridge type
\end{tabular}

${ }^{a}$ Statistics on traffic volume include large and small vehicles [37].

$$
C_{a b}=C_{1} \cdot x^{-b}
$$

where $C_{a b}$ is airborne salt concentration (mdd $\cdot \mathrm{NaCl}$ ); $C_{1}$ is $1 \mathrm{~km}$ equivalent airborne salt concentration ( $\mathrm{mdd} \cdot \mathrm{NaCl}) ; x$ is the distance from coastline $(\mathrm{km})$; and $b$ is the degree of distance attenuation $(b=0.6)$.

$C_{1}$ for areas B and $\mathrm{C}$ shown in Figure 4 is 1.174 and 0.072 , respectively [39]. Afterwards, empirical equation (5) [40] can be used to compute the chloride ion concentration that adheres to the surface of the concrete.

$$
C_{0}=-0.016 \times C_{a b}^{2}+C_{a b}+1.7\left(C_{a b} \leq 30\right),
$$

where $C_{0}$ is chloride ion concentration of concrete surface $\left(\mathrm{kg} / \mathrm{m}^{3}\right)$; and $C_{a b}$ is airborne salt concentration at the bridge location (mdd $\cdot \mathrm{NaCl}$ ).

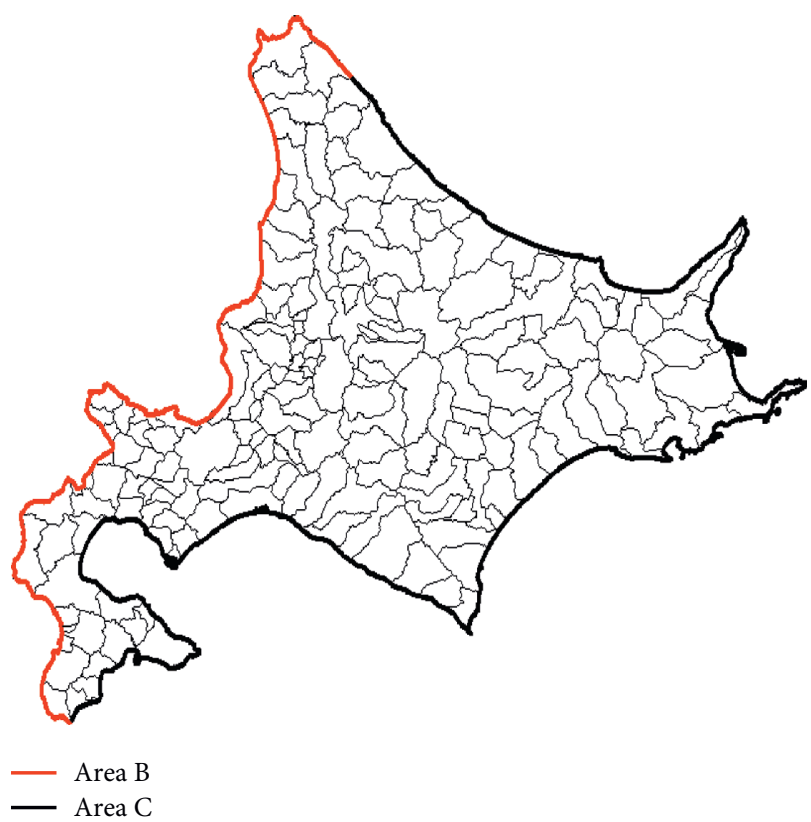

Figure 4: Salt damage area classification in Hokkaido.

4.1.2. Deterioration Grades. The deterioration grades are used to understand the conditions of the bridges. According to the inspection guidelines [8], the deterioration situations of bridges are categorized as follows: grade 1: healthy; grade 2: preventive maintenance required; grade 3 : early action required; and grade 4: emergency action required. The grades and corresponding descriptions are given in Table 2. Since grade 4 is considered a critical condition, meaning that these bridges have to be subjected to a repair timely, this philosophy results in the infrequent occurrence of grade 4 . Therefore, grade 3 is considered to be the upper threshold, because grade 3 indicates the necessity of early actions. This consideration prompted us to build a prediction model to predict the situation before an emergency occurs. The prediction model can be taken as a tool to distinguish seriously and nonseriously deteriorated bridge. Then, the seriously deteriorated bridge can be introduced to the detailed inspection and maintenance.

4.1.3. Data Characteristics Analysis. Twelve factors listed in Table 1 are investigated. Since different factors are measured on different ranges, values of these factors are necessary to be adjusted to a common scale to improve the efficiency of the training process. As listed in Table 3, all the factors were scaled within $0-1$. Years in service indicate the bridge age after it was built. Carbon dioxide refers to the concentration near the ground at the bridge location. Large-size car vehicles rate is calculated by equation (3). The type of bridge was excluded when establishing the ANN model, as it is irrational to represent different bridge types with a number. Although it is possible to build models for different types of bridges, the lack of sufficient data on each type of bridge limits this attempt. Discussions regarding the performance of the ANN model on different types of bridges are described in Section 4.2.2. 
TABLE 2: Inspection grades and corresponding conditions of bridges.

\begin{tabular}{lcc}
\hline Grade & Conditions & Descriptions \\
\hline 1 & $\begin{array}{c}\text { Healthy } \\
\text { Preventive action } \\
\text { required }\end{array}$ & Although the function of the structure is not hindered, it is desirable to take measures from the viewpoint \\
of preventive maintenance. & The function of the structure has interfered and measures should be taken as soon as possible. \\
3 & $\begin{array}{c}\text { Early action required } \\
\text { Emergency action } \\
\text { required }\end{array}$ & $\begin{array}{c}\text { The fondition in which the function of the structure has been or is likely to be impaired, and measures } \\
\text { should be taken urgently. }\end{array}$ \\
\hline
\end{tabular}

TABLE 3: Scaled values of each influencing factor.

\begin{tabular}{lccc}
\hline Factor & Original data range & \multicolumn{2}{c}{ After scaled } \\
& & Mean & 0.1347 \\
\hline Elevation $(\mathrm{m})$ & $-0.3 \sim 1,106$ & 0.0909 & 0.0844 \\
Bridge length $(\mathrm{m})$ & $2 \sim 1,433$ & 0.0444 & 0.1124 \\
Bridge width $(\mathrm{m})$ & $0.7 \sim 50$ & 0.2129 & 0.2008 \\
Years in service (years) & $1 \sim 84$ & 0.3833 & 0.2413 \\
Carbon dioxide (ppm) & $348.86 \sim 409.87$ & 0.3933 & 0.1376 \\
Chloride $\left(\mathrm{kg} / \mathrm{m}^{3}\right)$ & $0 \sim 17.3$ & 0.057 & 0.1786 \\
Large-size vehicles rate $(\%)$ & $0 \sim 62.6$ & 0.3076 & 0.1143 \\
Traffic volume (daily) & $0 \sim 56,874$ & 0.0946 & 0.2268 \\
Rainfall $(\mathrm{cm})$ & $776 \sim 1,491$ & 0.3617 & 0.2166 \\
Snowfall $(\mathrm{cm})$ & $12.0 \sim 1,263.8$ & 0.4493 & 0.1737 \\
Highest temperature $\left({ }^{\circ} \mathrm{C}\right)$ & $16 \sim 26$ & 0.7025 & 0.2361 \\
Lowest temperature $\left({ }^{\circ} \mathrm{C}\right)$ & $-11 \sim-3$ & 0.5851 & \\
\hline
\end{tabular}

\subsection{Prediction Model}

4.2.1. Model Establishment. The twelve factors in Table 3 and three deterioration grades were used to construct an ANN model. The optimal configuration for the ANN model was determined by trial and error. A higher accuracy was considered better performance of a prediction model. In the trial and error, various values were tested for each parameter [32], as listed in Table 4. Figure 5 depicts the nonlinear activation functions used in the trial and error. The softmax was applied between the hidden layer and the output layer. Relu and tanh were applied between the input and hidden layers.

Considering all combinations of the five parameters, 112 cases were tested. In each trial, stratified sampling was used to randomly divide the dataset into the training, testing, and validation subsets, which accounted for $70 \%, 15 \%$, and $15 \%$ of the maintenance database, respectively. Then each case was tested three times to obtain an average value to eliminate the variance. Afterwards, analysis of variance (ANOVA) analysis was applied to the testing results to examine the significance of each parameter on training/validation accuracy. A $p$ value of 0.05 or less is considered to have a significant influence on deterioration. The $p$ value summarized in Table 4 indicates that the learning rate and learning algorithm influence training/validation accuracy significantly. Looking through the testing results, the ANN produces higher accuracy when it has one hidden layer of ten neurons, has the activation function of Relu, and is trained with the adaptive moment estimation (Adam) algorithm with the learning rate of 0.01 .
The expected ANN consisted of a 3,368 $\times 13$ matrix as the inputs (including the bias) and a $3,368 \times 1$ matrix as the output. As the initial values of $W_{1}$ and $W_{2}$ (Figure 1) are randomly assigned during training, the predicted grades are usually inconsistent with the actual grades. The cross-entropy value expressed in equation (6) was used to calculate the error between the actual and the predicted grades. $M$ is the number of samples, $C$ is the number of classes, $\eta_{j}$ denotes the weight for class $j$ to deal with sample imbalance, and $p_{j}$ represents the probability that a sample belongs to class $j$. If a sample belongs to class $j, y_{j}=1$; otherwise $y_{j}=0$.

$$
\text { Cross entopy }=-\sum_{i=1}^{M} \sum_{j=1}^{C} \eta_{j} y_{j} \log \left(p_{j}\right) \text {. }
$$

The training and validation iterated 2000 times, and the error between the predicted value and the ground truth was evaluated by using the cross-entropy. As shown in Figure 6, the accuracy converges to approximately $65 \%$, and the error converges to approximately 0.2 . The accuracy and the error show that the established model is some distance from perfection. Future endeavors are necessary to improve the established model by actions described in Section 5.2.

4.2.2. Evaluation of the Prediction Model. The performance of the prediction model was evaluated using five metrics. The definitions in terms of these metrics were introduced in Figure 7 taking grade 1 as an example. TP, TN, FP, and FN refer to true positives, true negatives, false positives, and false negatives, respectively. Recall or true positive rate (TPR) is 
TABLE 4: Configuration test results.

\begin{tabular}{lccc}
\hline Parameters & Parameters & Training & $p$ value \\
\hline Number of hidden layers & & 0.5489 & 0.1154 \\
Number of hidden neurons & 1,2 & 0.4236 & 0.8454 \\
Learning rate & 5,10 & $0.0003^{\mathrm{a}}$ & $0.0038^{\mathrm{a}}$ \\
Learning algorithm & $0.01,0.001$ & $5.513 \times 10^{-14 a}$ & $8.490 \times 10^{-15 a}$ \\
Hidden activation function & SGD, RMSProp, Adagrad, & 0.4904 & 0.7622 \\
\hline
\end{tabular}

${ }^{a}$ Significant at $95 \%$ level of confidence.

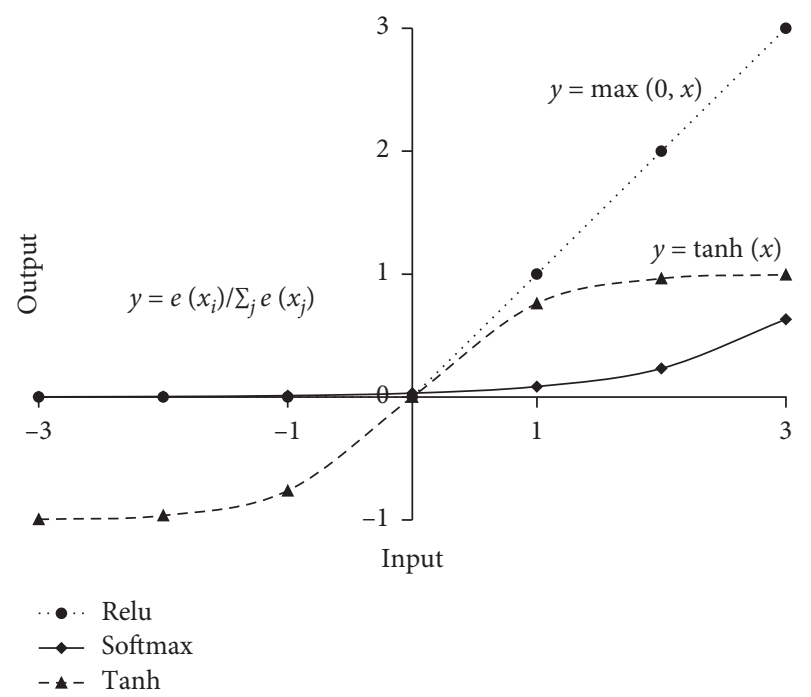

Figure 5: Nonlinear activation functions.

the ratio of correct predictions to all grade 1 bridges. Precision is the ratio of correct predictions to all predictions of grade 1 . The $F 1$ score is the harmonic mean of the recall and precision. The true negative rate (TNR) indicates the ratio of correct predictions on other grade bridges to the total number of other grade bridges. A model is considered better performance with higher values of these metrics. For details about these metrics, refer to Goodfellow et al. [32].

Figure 8 shows these metrics' values of the ANN model for the three deterioration grades. The results indicate that the established model has equivalent performance for the three deterioration grades. In addition, the model is applied to PC, RC, mixed, and steel bridges to evaluate the model's performance for corresponding type of bridge. Figure 9 shows that the model has equivalent performance regardless of the types of bridges. Although the established model is not yet perfect, the established model is unbiased for the different deterioration grades and different types of bridges.

4.3. Estimation of Factor Importance. Using the prediction model, successful predictions were made for 2,161 out of the 3,368 bridges. Therefore, a new database of 2,161 bridges was set up. The Shapley value and Sobol indices methods were then applied to perform the sensitivity analysis of each factor from the new database. The calculated sensitivities were normalized to show their relative importance, as indicated in
Figure 10. The five most important factors determined by the Sobol indices and Shapley value method are the same, although their values are different. Bridge length and years in service were determined to be the most important factors affecting the deterioration. In addition, traffic volume, lowest temperature, and chloride ions are the three external factors that influence deterioration, which is consistent with known findings. Therefore, the Shapley value method was considered a suitable solution to determine the contribution of factors on deterioration. Furthermore, the Shapley value method was applied to analyze the new database from other aspects.

\subsubsection{Distribution of Each Potentially Influencing Factor.} According to the Shapley value method, the estimated importance of all factors was calculated for each bridge. The estimated importance of all bridges separated by each factor is shown in Figure 11. The estimated importance indicates the relative importance of each factor for a bridge. A positive value represents that the factor will accelerate the deterioration, and negative value means that the factor will slow down the deterioration. Therefore, six factors (such as years in service, carbon dioxide concentration, and the chloride ion concentration) showed accelerated effects on deterioration. Other factors (such as elevation, bridge length, and bridge width, rate of the large-size vehicles, rainfall, and highest temperature) did not show consistent effects. Only the six factors with acceleration were discussed hereafter. In addition, Figure 11 revealed that the estimated importance of lowest temperature was negatively related to the measured value. The estimated importance of other five accelerated factors, such as years in service and traffic volume, showed positive correlations with the measured values.

4.3.2. Estimated Importance of Each Grade. To compare the estimated importance between different grades, the average importance of the six factors for its respective grade was computed, as shown in Figure 12. Of all the factors, years in service and traffic volume are the two most significant factors for all grades. Other factors have relatively fewer impacts. Since the service time of grade 1 bridges is generally short, environmental factors such as lowest temperature and chloride ions have not had a significant impact. Therefore, the traffic volume is the most significant factor. As time goes by, the importance gap between the years in service and the traffic volume traffic has narrowed, as shown in grade 2 . The results indicate that the relative importance of the years in 


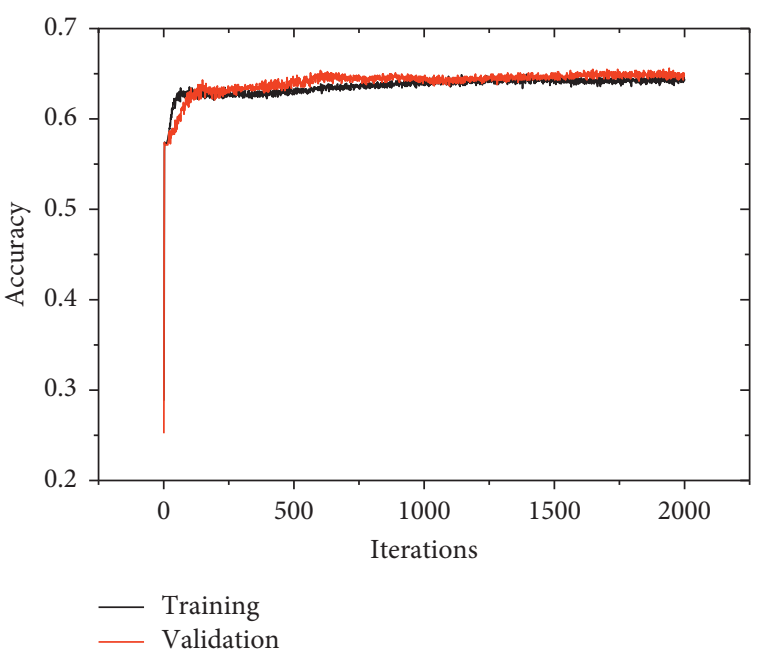

(a)

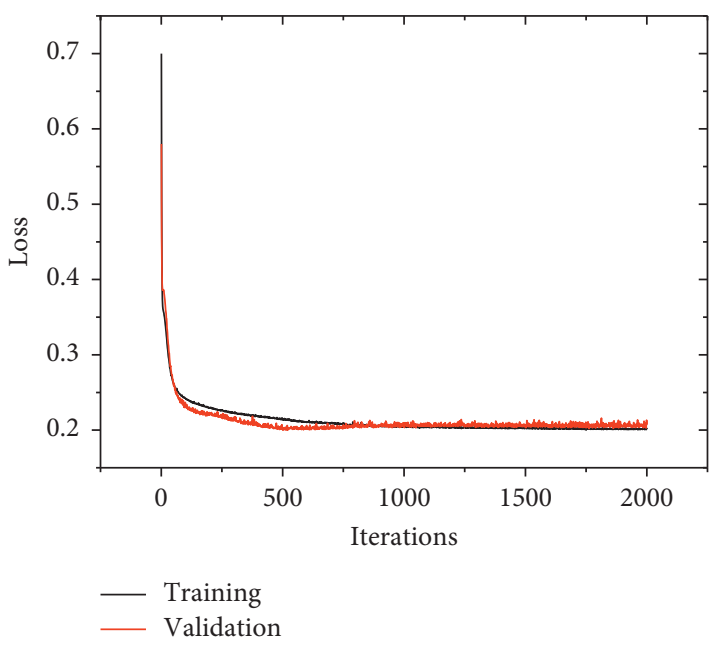

(b)

FIgURe 6: Loss and accuracy.

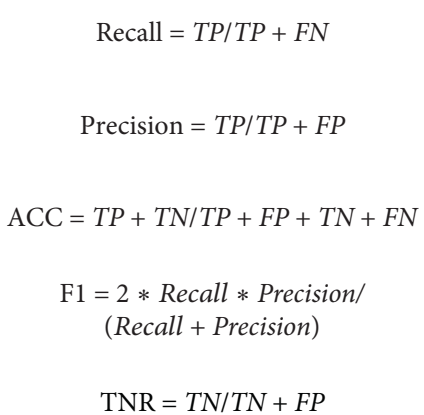

$\mathrm{TNR}=T N / T N+F P$

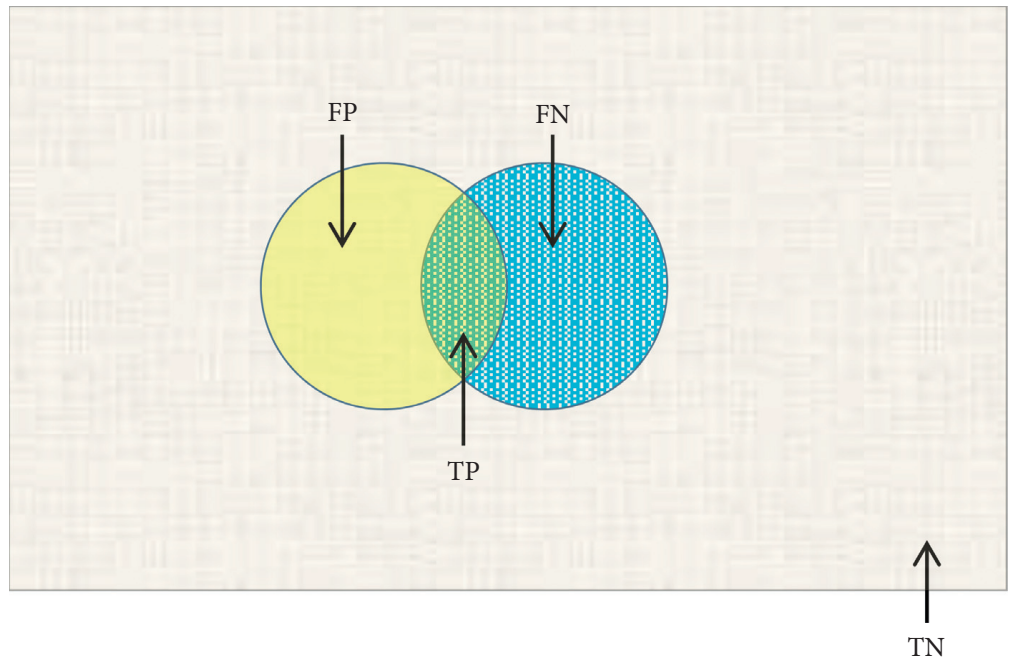

Gradel prediction

Grade1 truth

Other grades' truth

Figure 7: Performance evaluation metrics used in this study (taking grade 1 for example).

service has increased, and its impact on deterioration has also increased. Further, the importance of the years in service and the traffic volume is almost the same in grade 3 . In addition, the influences of the lowest temperature and the chloride are increased. Because the bridges in grade 3 have been in service for an average of more than 46 years, a relatively smaller importance of a factor does not mean the factor is unimportant, because the cumulative effects of these factors are not considered. Therefore, various factors should be comprehensively considered to provide recommendations for future maintenance. In addition, intervention is performed for some bridges, which is difficult to be considered in our methodology.
4.3.3. Estimated Importance of Different Structure Type. The deterioration differs for different types of bridges. To observe whether the estimated importance can reflect the differences caused by different types of bridges, the average estimated importance of all factors, broken down by structure types, is shown in Figure 13.

Similar to the findings in previous sections, the years in service and the traffic volume are the two most significant factors for all the bridge types. The distribution of these factors differs in different bridge types. For the PC bridges, other factors are less important relative to the years in service, as PC bridges are generally able to withstand harsher conditions and have better durability. Compared with the PC bridge, the importance 


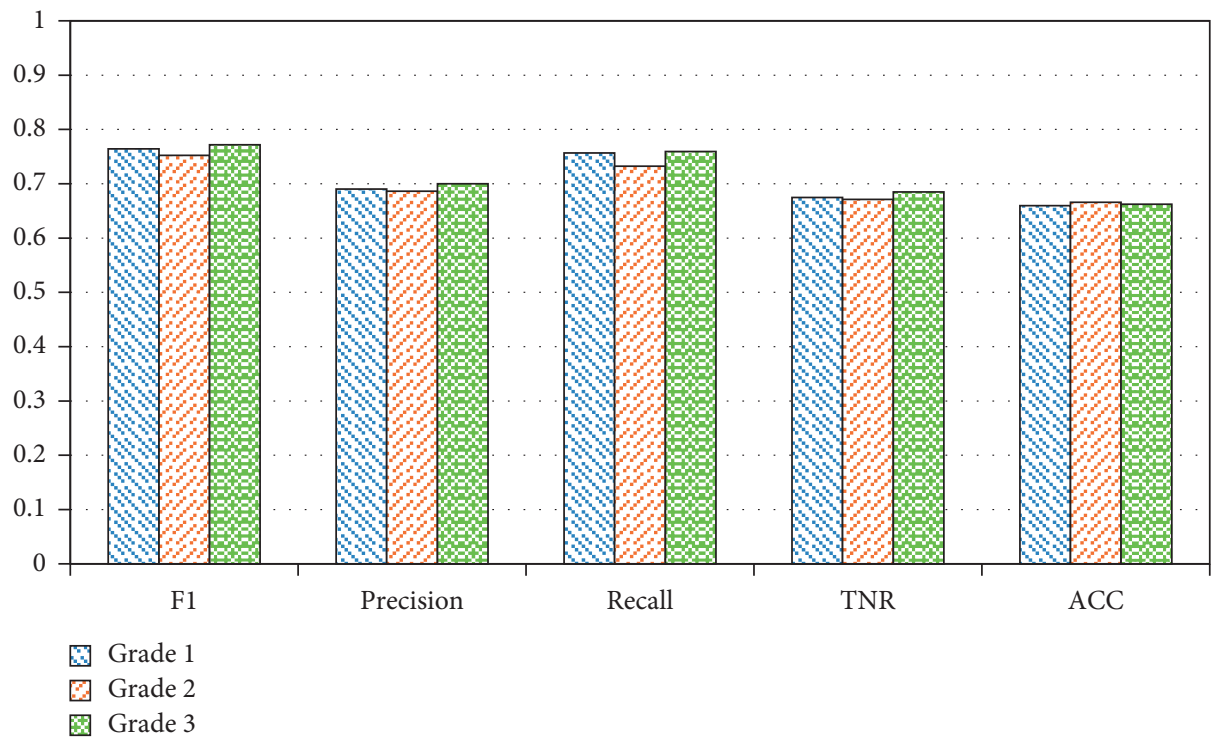

Figure 8: Performances of the established neural network for different grades.

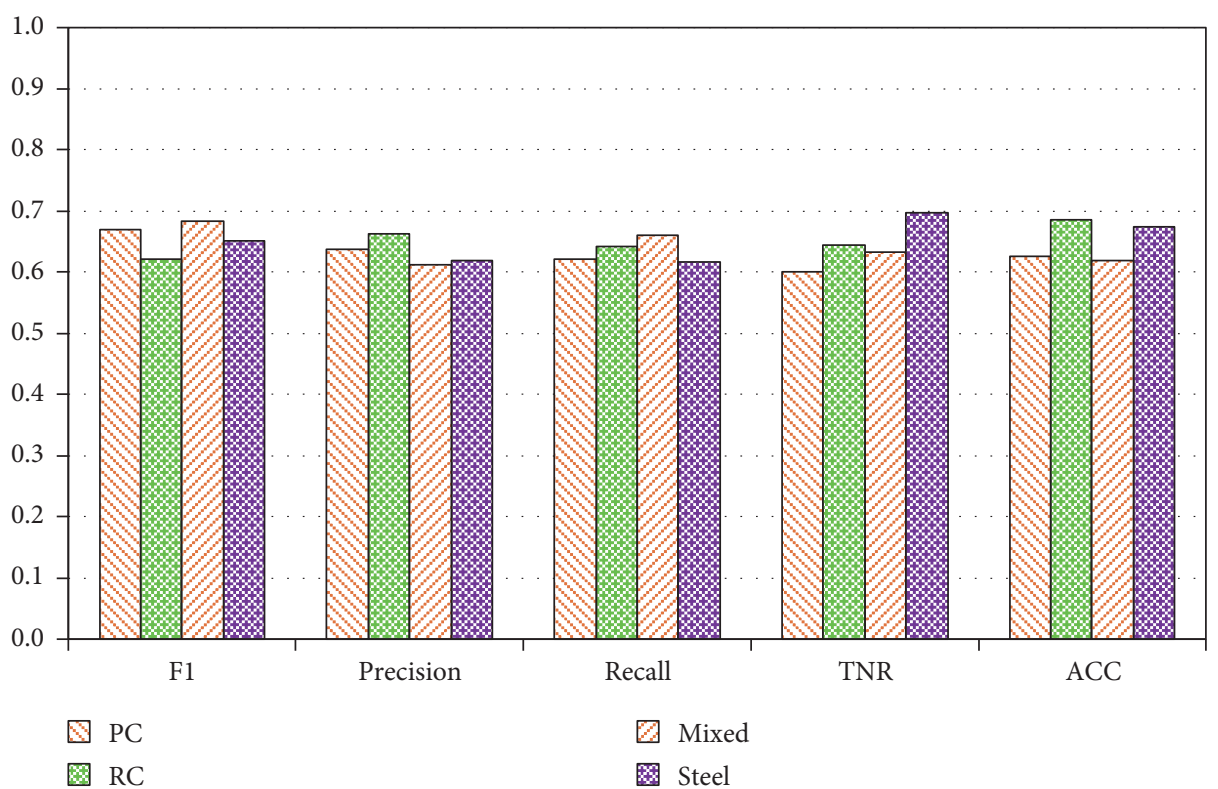

Figure 9: Performances of the established neural network for different types of bridges.

of the RC bridge is more uniformly distributed, because RC bridges are more susceptible to loads (traffic volume) and environmental factors (lowest temperature and chloride ion). The mixed bridge is almost in the middle of the PC bridge and $\mathrm{RC}$ bridge. For steel bridges, the importance of traffic volume is increased because the average daily traffic volume of this type of bridge in the applied database is usually more than 10,000 and usually accommodates many large vehicles (3337 vehicles/day).

As a result, the bridge type is determined to be another reason to lead to differences in deterioration. As for other researcher's findings [27], materials and surrounding environments should be the most significant factors. Since the database regarding the bridge materials is unavailable, this study did not consider the differences induced by the materials. In terms of the environment, bridges will be divided into two groups depending on the bridge is located in coastal or noncoastal regions, which will be discussed later.

\subsubsection{Estimated Importance of Different Environments.} Since bridges in coastal areas are exposed to extremely severe environments for materials [41], the environments of bridges were divided into coastal and noncoastal regions according to bridge locations, and the average importance for these two environments was calculated, as shown in Figure 14. For bridges in noncoastal areas, the distribution of these factors is the same as in the previous sections, except that the estimation for chloride ion is zero because deicing 


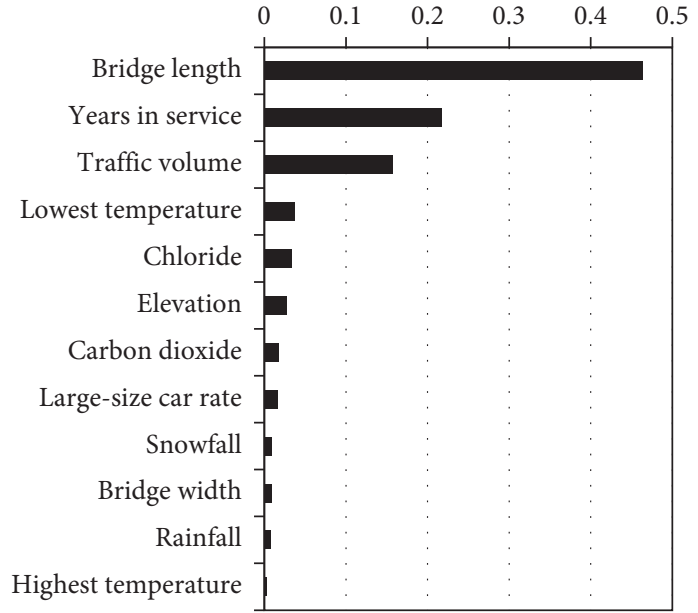

(a)

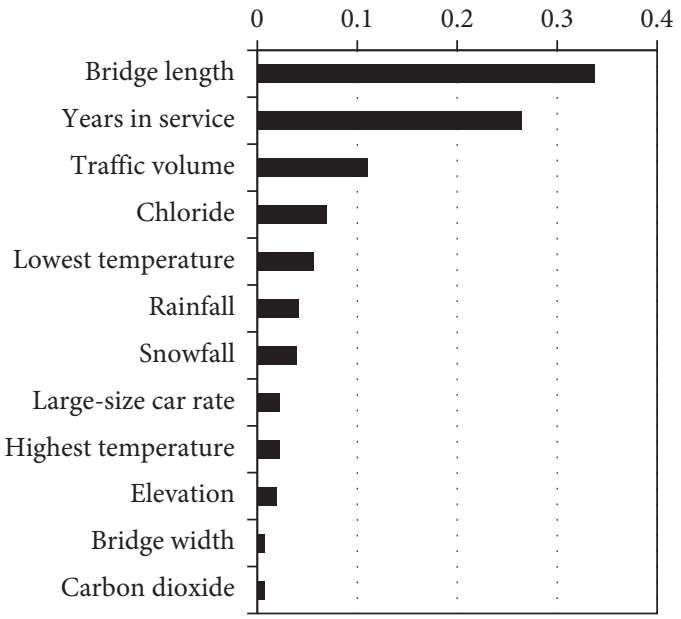

(b)

FIgURE 10: Sensitivity analysis by (a) Shapley indices and (b) Sobol value methods.

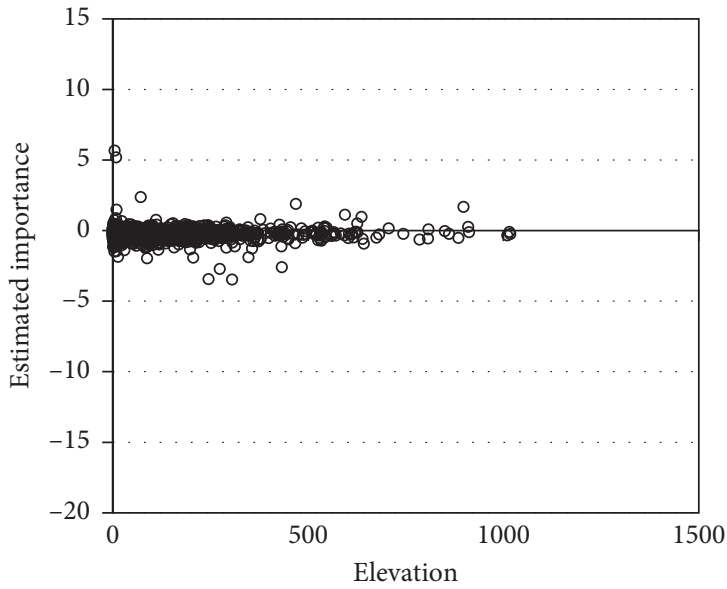

(a)

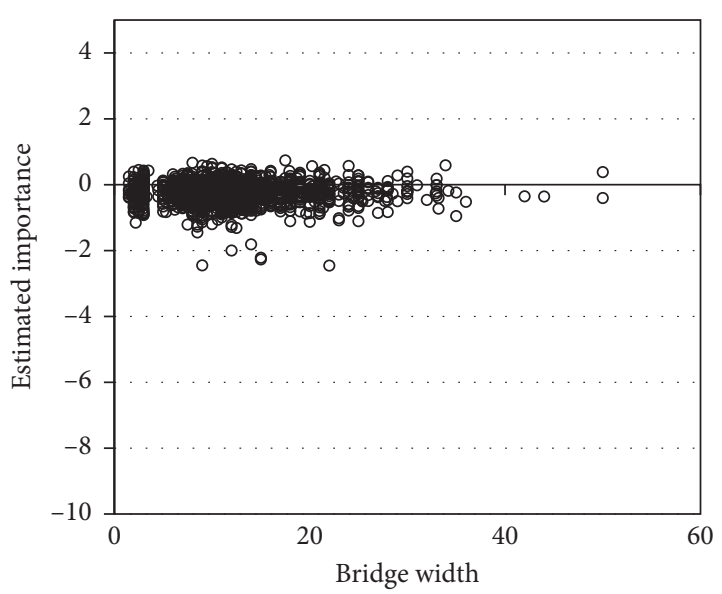

(c)

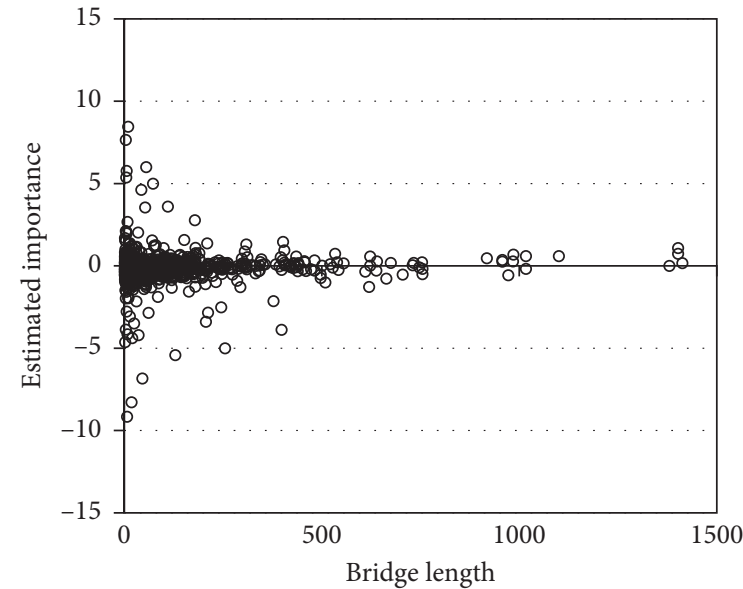

(b)

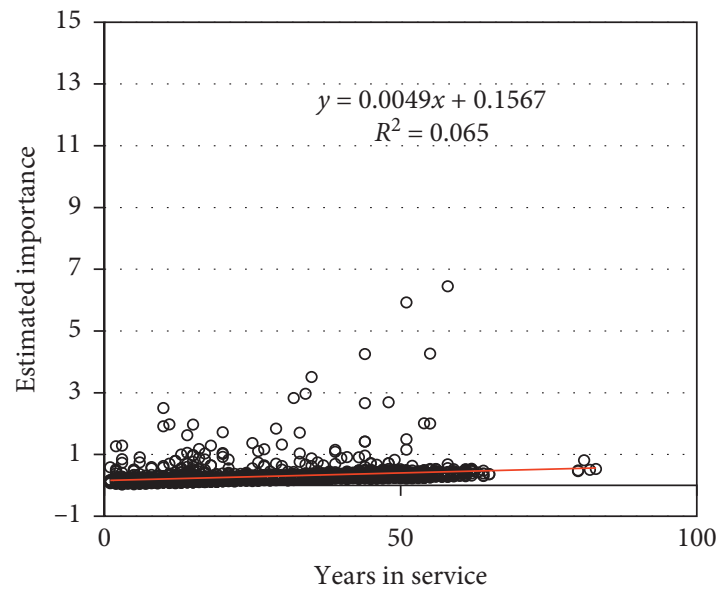

(d)

Figure 11: Continued. 


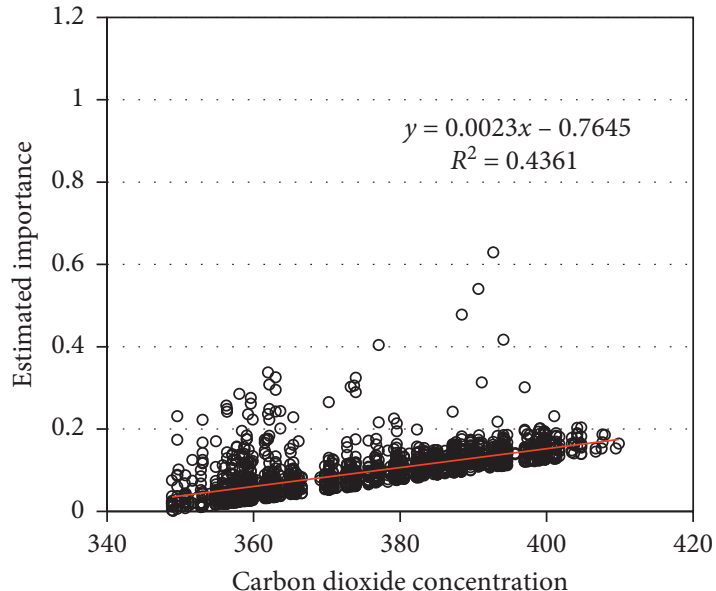

(e)

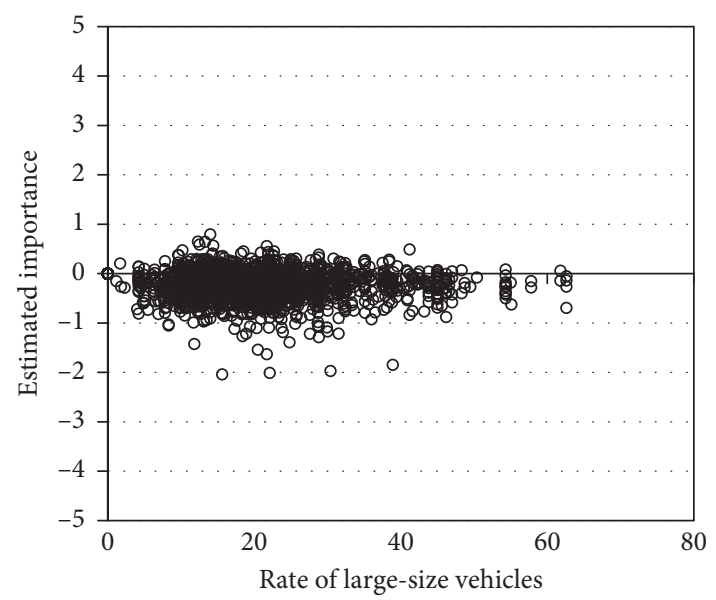

(g)

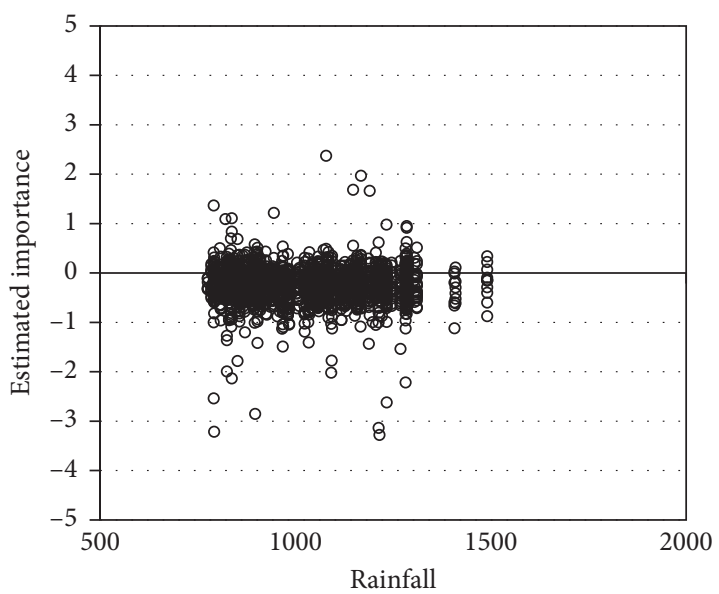

(i)

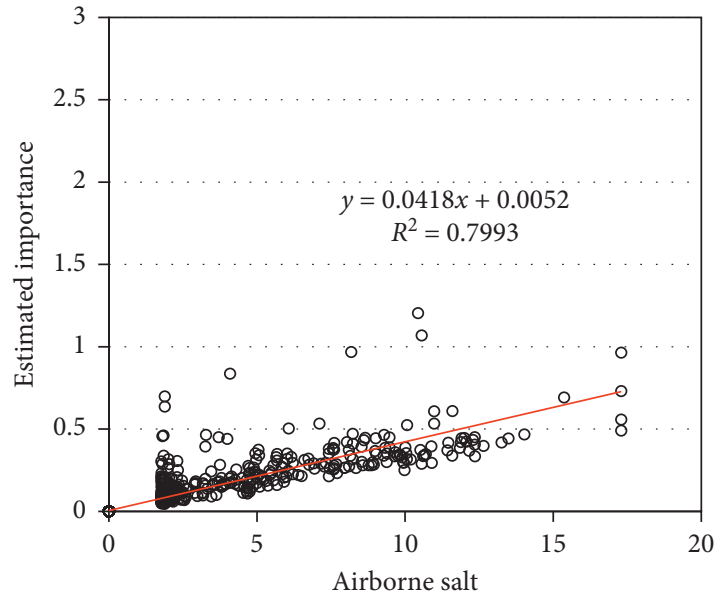

(f)

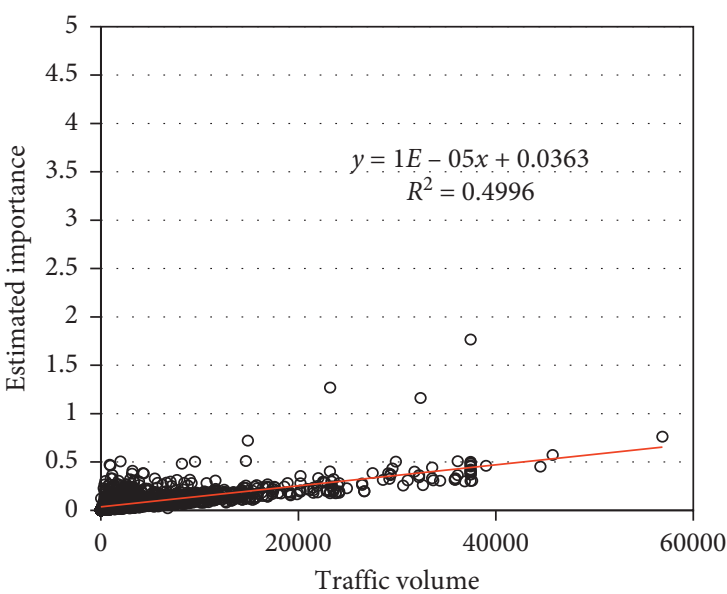

(h)

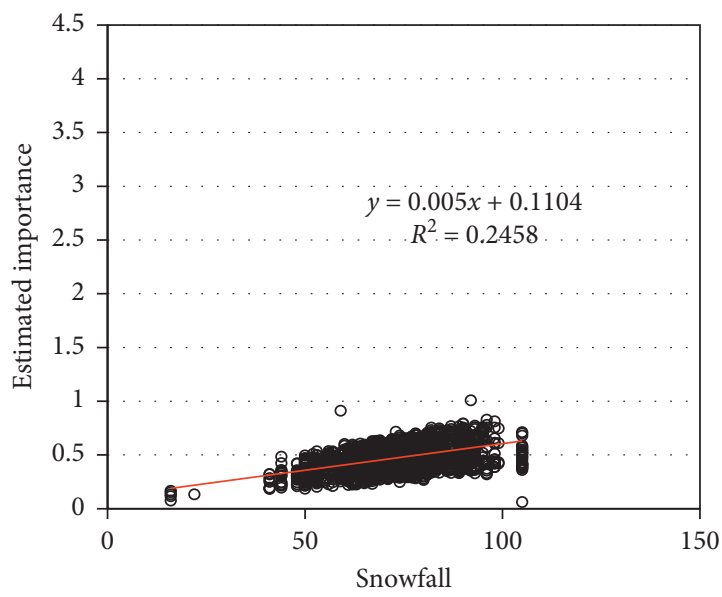

(j)

Figure 11: Continued. 


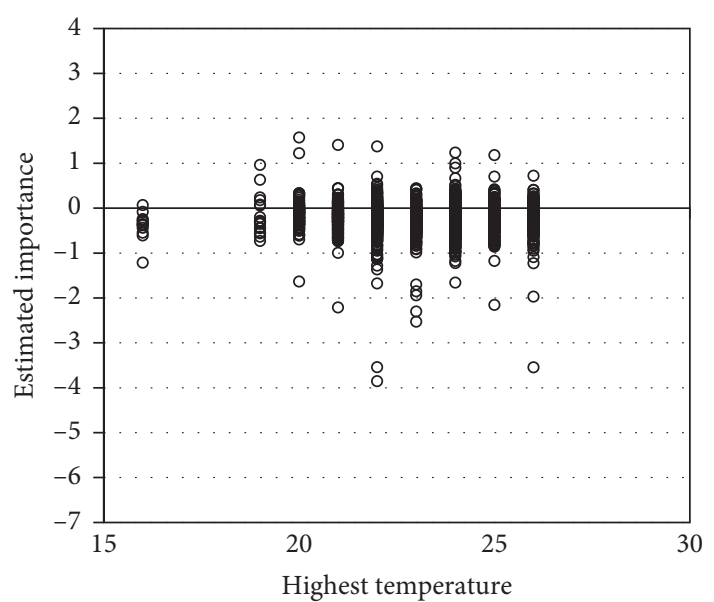

(k)

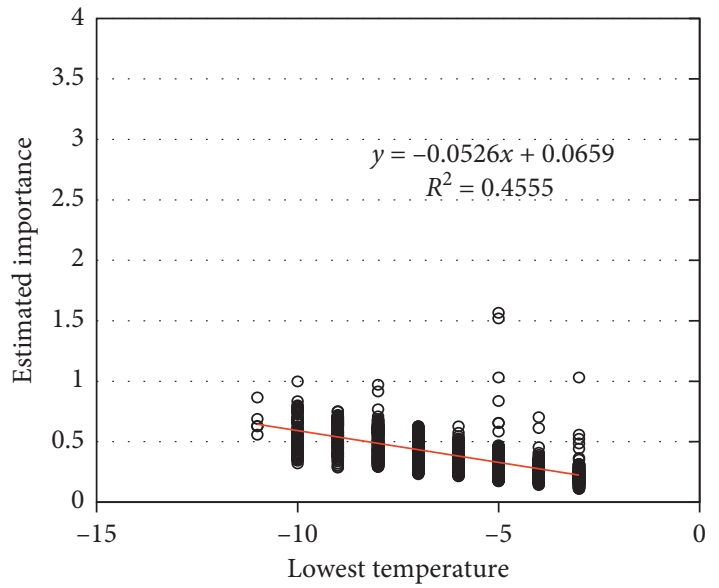

(1)

FIGURE 11: Estimated importance of each factor for all bridges.

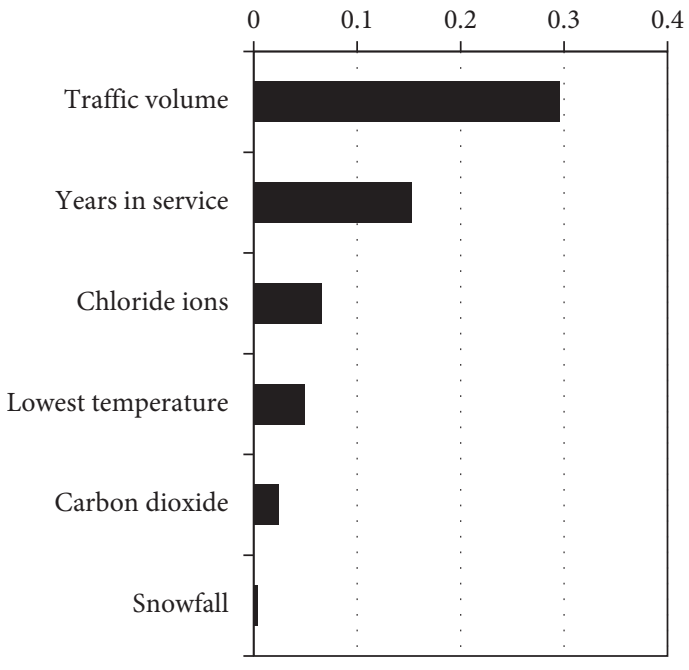

(a)

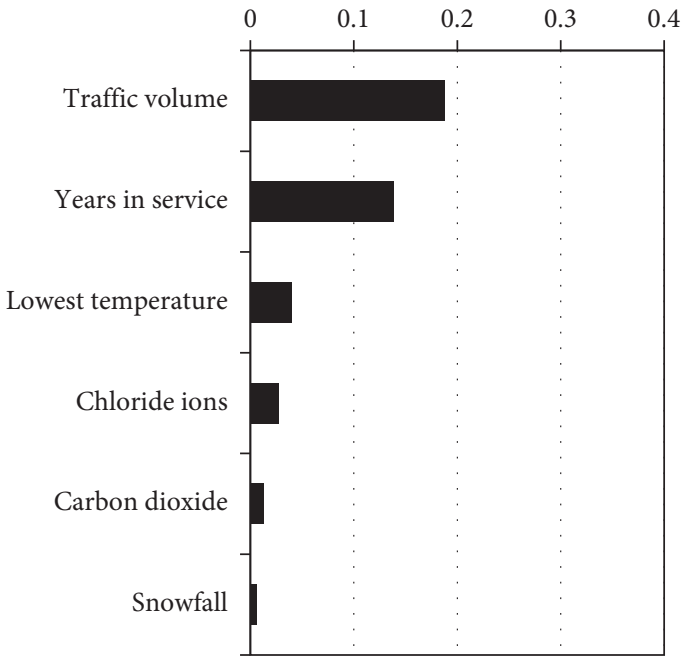

(b)

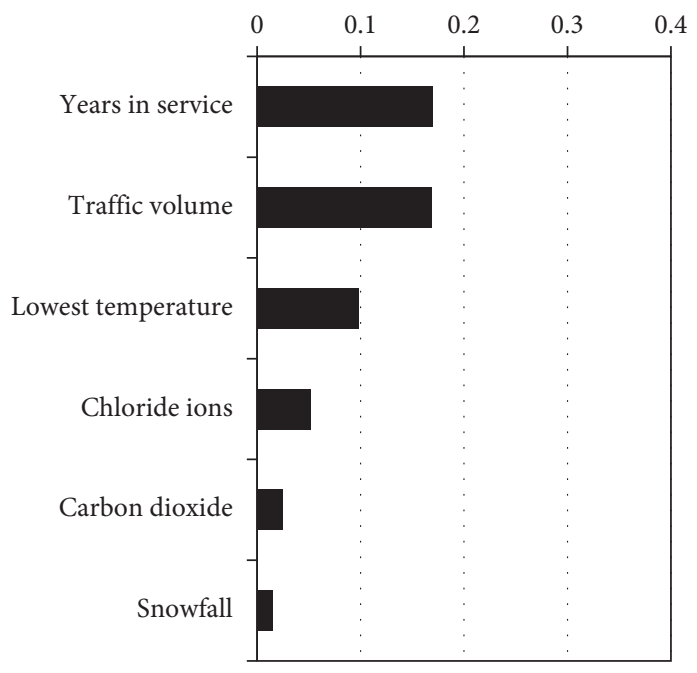

(c)

FIGURE 12: Average importance of each factor, broken down by grade. (a) Grade 1, (b) grade 2, (c) grade 3. 


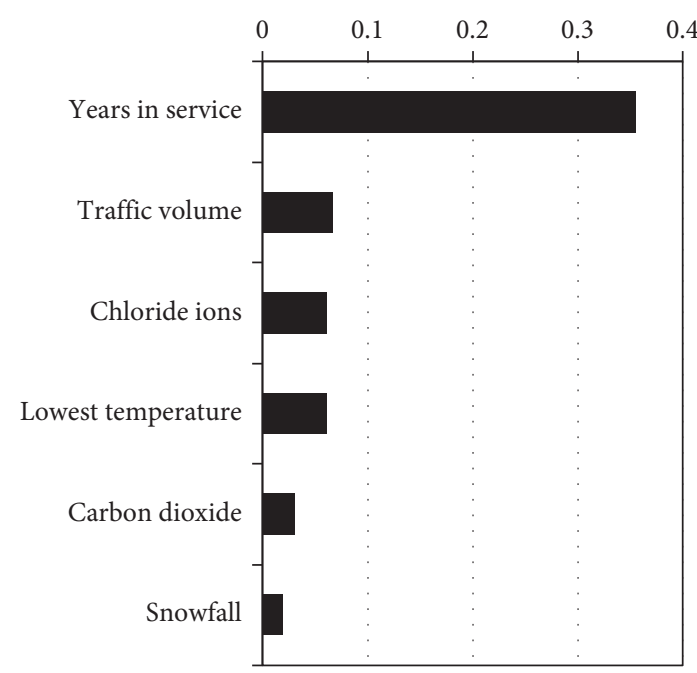

(a)

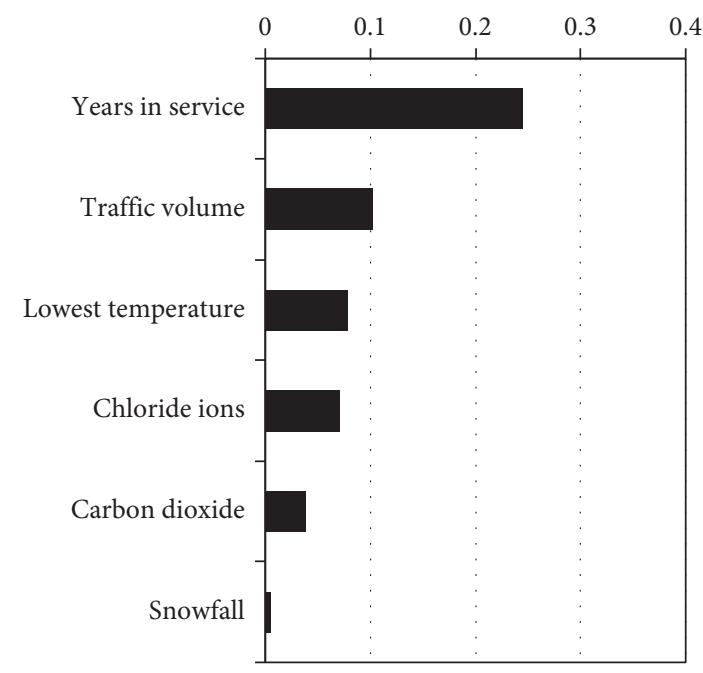

(c)

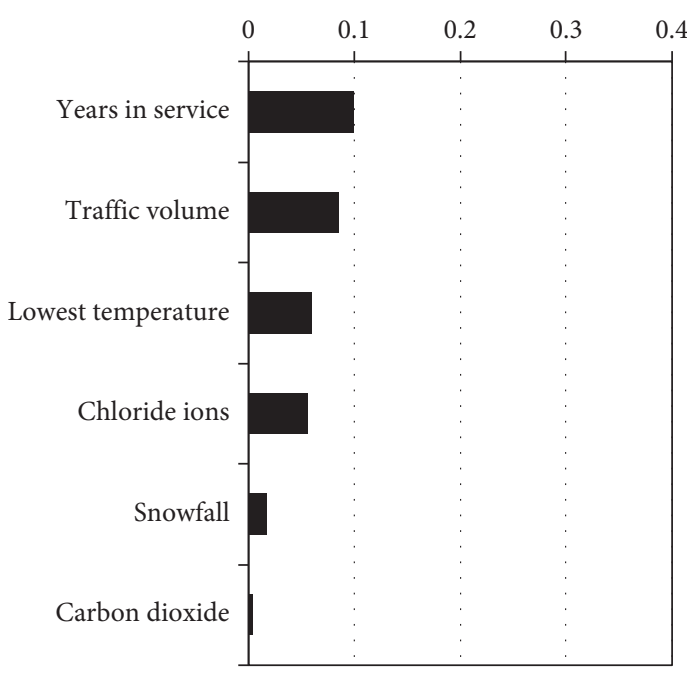

(b)

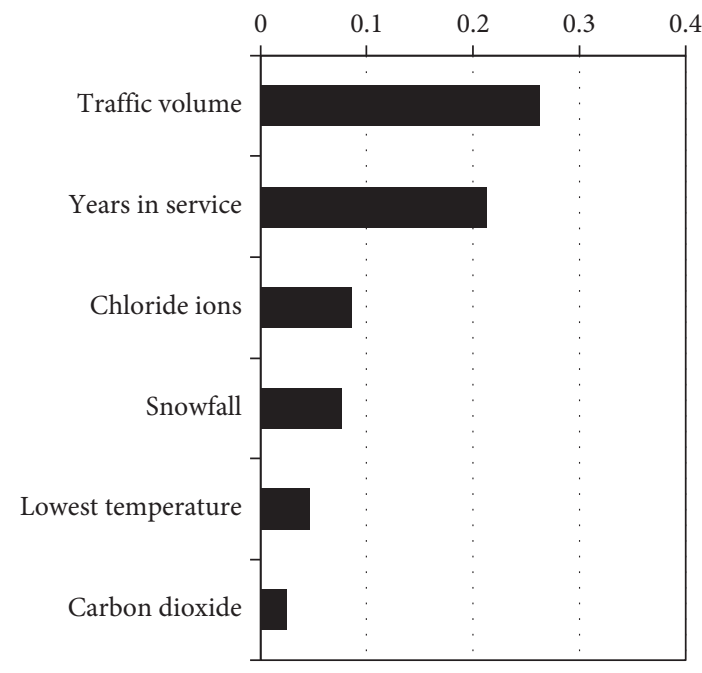

(d)

FIGURE 13: Average importance of each factor, broken down by the bridge type. (a) PC, (b) RC, (c) mixed, and (d) steel.

salt was not considered. Compared with noncoastal conditions, the impact of chloride ions in coastal region is greatly increased. In addition, the traffic volume and lowest temperature were identified two crucial factors regardless of the coastal region or noncoastal region.

The results indicated that the bridges in coastal areas are more strongly affected by airborne salt, and the estimated importance of the sensitivity analysis meets reality. In summary, there is a reasonable agreement between the estimated factors with accelerated effects and factors already known to affect the deterioration. Therefore, the sensitivity analysis can identify factors influencing deterioration and can calculate their relative importance.

\section{Discussions}

The practical usage of the proposed methodology was described using two bridges as examples. In addition, the limitations of this study were discussed.
5.1. Formulation of Maintenance Strategy. In practical, the established ANN can be applied to obtain the degradation progress of the targeted bridges to support the detailed inspection and maintenance, as described by taking bridges $\mathrm{A}$ and $\mathrm{B}$ as examples (Table 5). Bridges $\mathrm{A}$ and $\mathrm{B}$ were inspected in 2016 and 2015, respectively, and both of them were assessed to be grade 1 . Bridges A and B have been in service for twelve years and fifty years, respectively. By updating the values of the factors listed in Table 5, the deterioration situation of the bridge can be predicted through the prediction model. As a result, the deterioration progresses of bridges $\mathrm{A}$ and $\mathrm{B}$ over time are shown in Figure 15. The results reveal that bridge $A$ will stay at grade 1 for a long time, while bridge B will degrade to grade 3 soon.

Figure 16 shows the estimated importance of each factor for bridges A and B. The results show that the years in service, chloride ions, and traffic volume are the three most significant factors for these bridges. The other three factors have relatively fewer influences. 


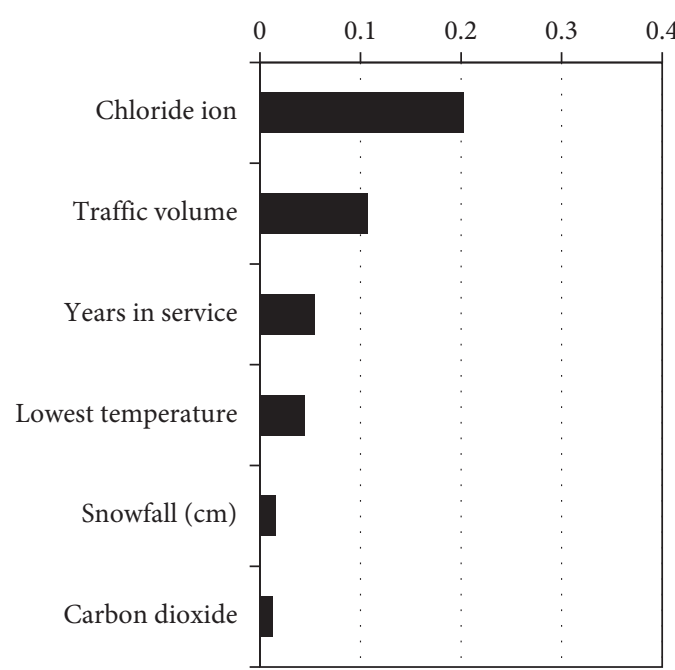

(a)

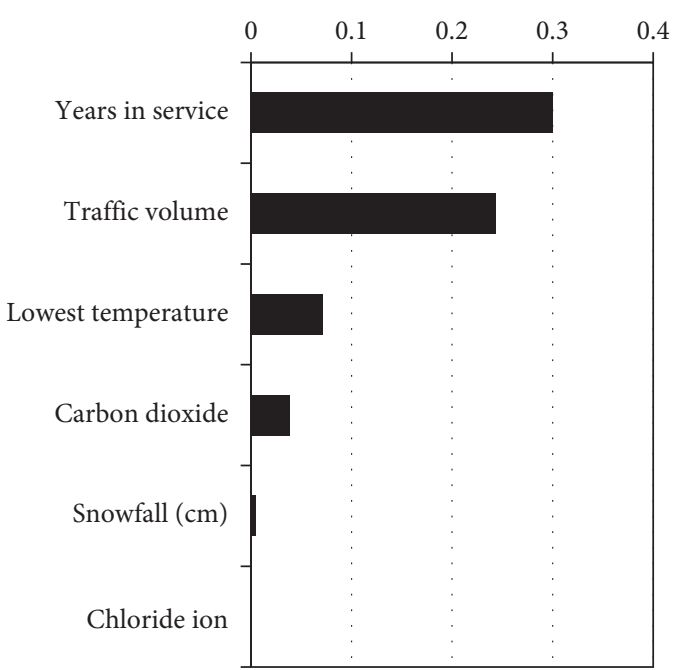

(b)

FIgURE 14: Average importance of each factor, broken down by (a) coastal region and (b) noncoastal region.

TABLE 5: Information of bridges A and B.

\begin{tabular}{lcc}
\hline & Bridge A & Bridge B \\
\hline Elevation $(\mathrm{m})$ & 3 & 2.9 \\
Bridge length $(\mathrm{m})$ & 35 & 12.54 \\
Bridge width $(\mathrm{m})$ & 9.5 & 11 \\
Years in service (years) & 12 & 50 \\
Carbon dioxide (ppm) & 393.32 & 356.38 \\
Chloride $\left(\mathrm{kg} / \mathrm{m}^{3}\right)$ & 2.2 & 2 \\
Large-size vehicles rate (\%/day) & 15.3 & 20.3 \\
Traffic volume (vehicles/day) & 1057 & 13233 \\
Rainfall $(\mathrm{cm})$ & 954 & 1060 \\
Snowfall $(\mathrm{cm})$ & 345.77 & 635.60 \\
Highest temperature $\left({ }^{\circ} \mathrm{C}\right)$ & 19 & 24 \\
Lowest temperature $\left({ }^{\circ} \mathrm{C}\right)$ & -3 & -3 \\
\hline
\end{tabular}

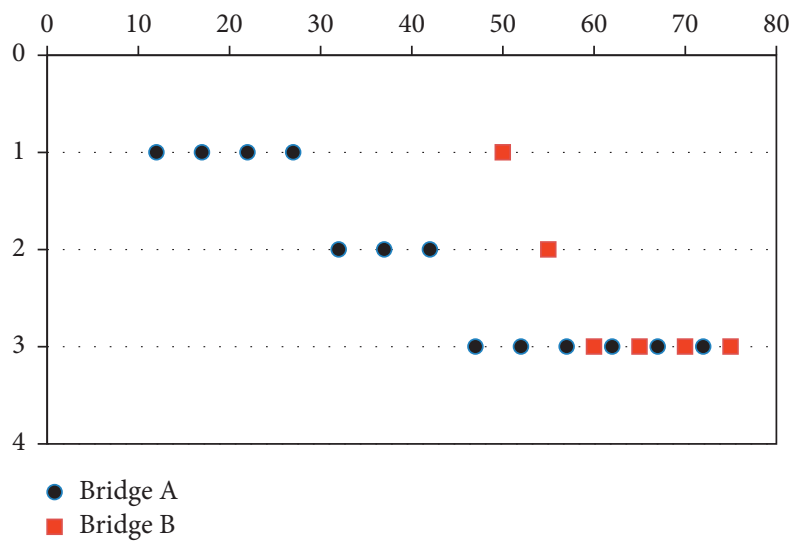

Figure 15: Prediction of the deterioration grades for bridges A and $\mathrm{B}$.

However, the estimated importance for the two bridges shows significant differences. For bridge A, the most significant factor is the traffic volume; the other factors are relatively lower. Because bridge A has been in service for only 12 years, the natural degradation of the materials is not obvious, and the impact of carbonation and chloride ion is still tiny. Considering bridge A will remain in grade 1 for the next 20 years, no special maintenance is necessary for bridge $A$ in ten years. As bridge B has been in service for 50 years and has recently accommodated many large-size vehicles, the lowest temperature, traffic volume, and chloride ion have all had essential impacts except for years in service. In addition, the deterioration of bridge $B$ will worsen from grade 1 to 3 soon, according to the deterioration curve in Figure 15. Therefore, bridge $\mathrm{B}$ should be introduced to the detailed inspection and fully maintained in five years. The results indicate that even though the deterioration of bridge A and bridge B are both being grade 1 , the calculated relative importance of all factors is different. Obviously, the estimated relative importance can reflect the bridge's properties and the reasons for deterioration. Additionally, further inspection and corresponding maintenance strategies can be set up.

5.2. Limitations. In this study, a methodology was proposed to predict deterioration and analyze the reasons for deterioration. The prediction model does not provide quantitative results for the deterioration situation of the bridge, but 


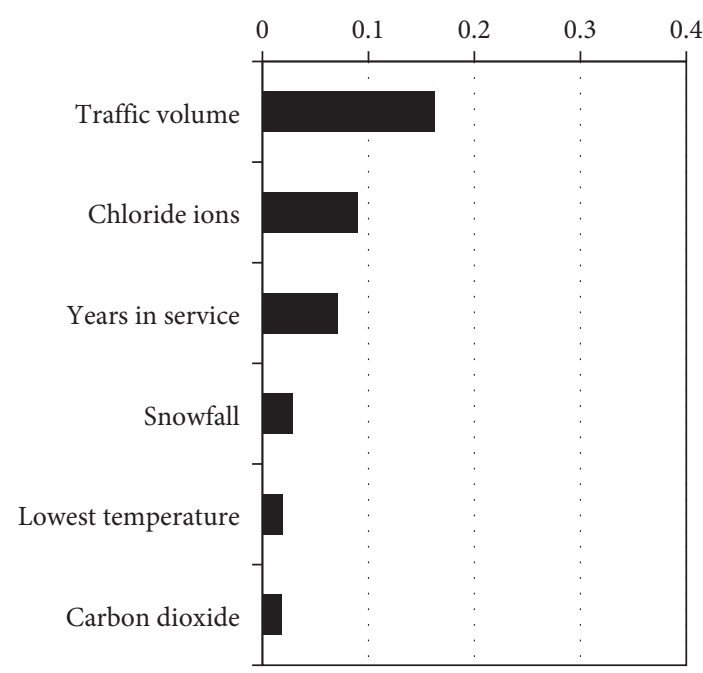

(a)

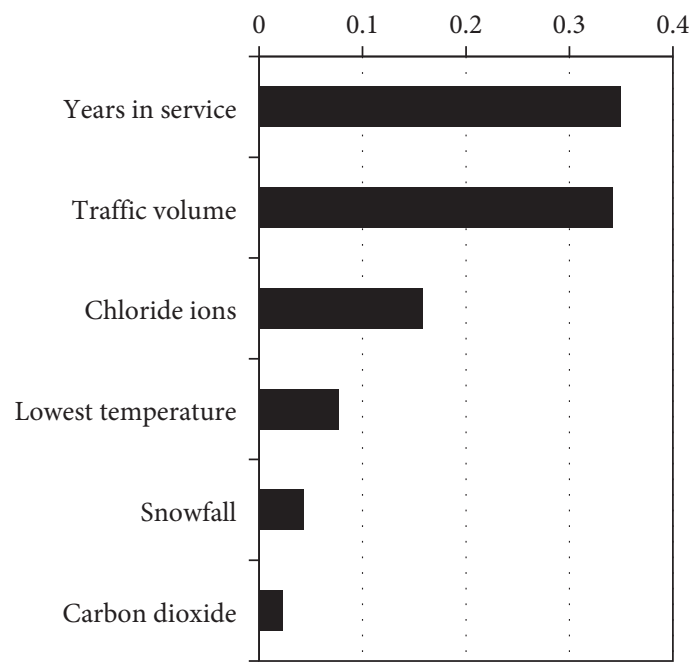

(b)

FIGURE 16: Estimated importance value for (a) bridge A and (b) bridge B.

it can be used as a supplementary tool in the preliminary inspection stage to distinguish seriously and nonseriously deteriorated bridges. Then, the seriously deteriorated bridge can be introduced to the detailed inspection and postmaintenance. Although the methodology is verified by a practical database, this study should still be regarded as an initial and ideal attempt. In this attempt, the bridge's specific materials was not considered in the deterioration predictions. For this reason, the analyzed results deviate from the practical situation to some extent. In addition, several other factors are associated with deterioration but are not considered, such as the application of deicing salt, design strength, and intervention histories. Furthermore, the deterioration differences in various components of the same bridge were not taken into account.

Because the applied database excludes bridges in grade 4 , the established model can only predict the deterioration situation of grade 3 and before. These deficiencies will encourage us to collect more data for improving future work. In assessing a factor's importance, the Shapley value method considers all possible influencing factors. But sometimes, the calculated importance is unreasonable because Shapley value method does not satisfy rational constraints. Since the deterioration grade is evaluated subjectively, the grades are not quantitatively relating to the deterioration situation. This leads to a bias in the calculated importance.

Future attempts could focus on collecting more relevant factors for improved interpretations of factors that contribute to deterioration. Another future improvement will be to modify the prediction and sensitivity analysis model, the integration of more high-quality data, and the quantitation of the deterioration grades.

\section{Conclusions}

This study proposed a methodology to integrate an artificial neural network (ANN) with sensitivity analysis method. The
ANN was performed to predict deterioration to determine further inspection and maintenance timing. The sensitivity analysis method offered insights into the prediction model to understand the reasons for deterioration. From the verification results using an inspection database, the following conclusions can be drawn:

(1) The combination of an ANN and sensitivity analysis method can effectively avoid the uncertainty of factors on deterioration to some extent. Therefore, the methodology can make predictions and understand factors affecting the predictions with the importance list of all influencing factors.

(2) An ANN was established considering twelve influencing factors as inputs and three deterioration grades as outputs. The established ANN obtained an accuracy of about $65 \%$. Although the model's performance is not yet perfect, the predictability is still acceptable considering the complexity of the existing bridges. In addition, the model has equivalent performance for three deterioration grades and four types of bridges. Practically, the deterioration progress of a bridge can be formulated to determine the detailed inspection and maintenance timing.

(3) The Sobol indices and Shapley value methods can identify the reasons for deterioration by performing the sensitivity analysis, and they show consistency for the five most important factors. This is an important step towards applying the neural network to practical maintenance because the sensitivity analysis converts abstract and inexplicable predictions into the relative importance of factors that are easy for engineers to understand and act on.

(4) From the estimated importance of factors, six factors were identified that would accelerate deterioration. They are years in service, carbon dioxide concentration, chloride ion concentration, traffic volume, 
snowfall, and lowest temperature. In addition, the analysis revealed that the bridge type is another significant reason to induce deterioration differences. The truth that the significant differences for bridges between coastal and noncoastal areas are the airborne salt was also revealed in the sensitivity analysis. The relative importance list of factors offers engineers the possibility of figuring out factors that significantly affect deterioration and formulating interventions accordingly for a specific bridge.

In the future, endeavors will be enforced to solve the limitations described in Section 5.2 to improve the performance of the proposed methodology.

\section{Data Availability}

The data used in the present study can be made available upon request from the authors.

\section{Conflicts of Interest}

The authors declare that they have no conflicts of interest.

\section{References}

[1] C. Andrade, "Propagation of reinforcement corrosion: principles, testing and modelling," Materials and Structures, vol. 52, no. 1, p. 2, 2019.

[2] C. Stenström, P. Norrbin, A. Parida, and U. Kumar, "Preventive and corrective maintenance - cost comparison and cost-benefit analysis," Structure and Infrastructure Engineering, vol. 12, no. 5, pp. 603-617, 2016.

[3] D. E. Rumelhart, G. E. Hinton, and R. J. Williams, "Learning representations by back-propagating errors," Nature, vol. 323, no. 6088, pp. 533-536, 1986.

[4] Z. Zhang, M. W. Beck, and M. W. Beck, "Opening the black box of neural networks: methods for interpreting neural network models in clinical applications," Annals of Translational Medicine, vol. 6, no. 11, p. 216, 2018.

[5] S. M. Lundberg, B. Nair, and M. S. Vavilala, "Explainable machine-learning predictions for the prevention of hypoxaemia during surgery," Nature Biomedical Engineering, vol. 2, no. 10, pp. 749-760, 2018.

[6] A. B. Owen, "Sobol' indices and Shapley value," SIAM/ASA Journal on Uncertainty Quantification, vol. 2, no. 1, pp. 245-251, 2014.

[7] I. M. Sobol, "Global sensitivity indices for nonlinear mathematical models and their Monte Carlo estimates," Mathematics and Computers in Simulation, vol. 55, no. 1-3, pp. 271-280, 2001.

[8] MLIT, "Guidelines for regular inspection of road bridges, MLIT,” 2018, https://www.mlit.go.jp/road/sisaku/yobohozen/ tenken/yobo4_1.pdf.

[9] S. Lee and N. Kalos, "Non-destructive testing methods in the U.S. for bridge inspection and maintenance," KSCE Journal of Civil Engineering, vol. 18, no. 5, pp. 1322-1331, 2014.

[10] Y. Jeong, W. Kim, I. Lee, and J. Lee, "Bridge inspection practices and bridge management programs in China, Japan, Korea, and U.S," Journal of Structural Integrity and Maintenance, vol. 3, no. 2, pp. 126-135, 2018.

[11] A. Al-Hussein, "Estimating bridge deterioration age using artificial neural networks," https://bspace.buid.ac.ae/handle/
1234/1128 Doctoral dissertation, The British University in Dubai, 2017.

[12] W. Hyman, D. Hughes, and T. Dobson, "The least cost mix of bridge replacement and repair work on wisconsin's state highways over time-a computer simulation," Technical Rep., WisDOT, Madison, WI, USA, 1983.

[13] G. Busa, M. Ben-Akiva, and O. Buyukozturk, Modeling Concrete Deck Deterioration, Department of Civil Engineering, Massachusetts Institute of Technology, Cambridge, MA, USA, 1985.

[14] A. Abu-Tair, C. McParland, J. Lyness, and A. Nadjai, "Predictive models of deterioration rates of bridges using the factor method based on historic inspection data," in Proceedings of the 9th International Conference on Durability of Building Materials and Components (DBMC), Brisbane, Australia, March 2002.

[15] N.-F. Pan, T.-C. Lin, and N.-H. Pan, "Estimating bridge performance based on a matrix-driven fuzzy linear regression model," Automation in Construction, vol. 18, no. 5, pp. 578-586, 2009.

[16] J. Kim, N. Gucunski, and K. Dinh, "Deterioration and predictive condition modeling of concrete bridge decks based on data from periodic NDE surveys," Journal of Infrastructure Methodologys, vol. 25, no. 2, Article ID 04019010, 2019.

[17] Y. Jeong, W. Kim, I. Lee, and J. Lee, "Bridge service life estimation considering inspection reliability," KSCE Journal of Civil Engineering, vol. 21, no. 5, pp. 1882-1893, 2017.

[18] A. K. Agrawal, A. Kawaguchi, and Z. Chen, "Deterioration rates of typical bridge elements in New York," Journal of Bridge Engineering, vol. 15, no. 4, pp. 419-429, 2010.

[19] L. Li, L. Sun, and G. Ning, "Deterioration prediction of urban bridges on network level using Markov-chain model," Mathematical Problems in Engineering, vol. 2014, Article ID 728107, 10 pages, 2014.

[20] N. K. W. Wellalage, T. Zhang, and R. Dwight, "Calibrating Markov chain-based deterioration models for predicting future conditions of railway bridge elements," Journal of Bridge Engineering, vol. 20, no. 2, Article ID 04014060, 2015.

[21] M. Mauch and S. Madanat, "Semiparametric hazard rate models of reinforced concrete bridge deck deterioration," Journal of Infrastructure Systems, vol. 7, no. 2, pp. 49-57, 2001.

[22] L. S. Lee, "Rehabilitation and service life estimation of bridge superstructures," in Service Life Estimation and Extension of Civil Engineering StructuresWoodhead Publishing, Cambridge, England, 2011.

[23] R. S. Adhikari, O. Moselhi, and A. Bagchi, "Image-based retrieval of concrete crack properties for bridge inspection," Automation in Construction, vol. 39, pp. 180-194, 2014.

[24] O. A. Hodhod and H. I. Ahmed, "Developing an artificial neural network model to evaluate chloride diffusivity in high performance concrete," HBRC Journal, vol. 9, no. 1, pp. 15-21, 2013.

[25] I. Mikami, S. Tanaka, and A. Kurachi, "Expert system with learning ability for retrofitting steel bridges," Journal of Computing in Civil Engineering, vol. 8, no. 1, pp. 88-102, 1994.

[26] J. O. Sobanjo, "A neural network approach to modeling bridge deterioration," in Computing in Civil Engineering, pp. 623-626, ASCE, Reston, VA, USA, 1997.

[27] Y.-H. Huang, "Artificial neural network model of bridge deterioration," Journal of Performance of Constructed Facilities, vol. 24, no. 6, pp. 597-602, 2010.

[28] S. Lim and S. Chi, "Bridge damage prediction using deep neural network," Computing in Civil Engineering 2019, Smart 
Cities, Sustainability, and Resilience, American Society of Civil Engineers, Reston, VA, USA, pp. 219-225, 2019.

[29] R. Caruana, Y. Lou, J. Gehrke, P. Koch, M. Sturm, and N. Elhadad, "Intelligible models for HealthCare," in Proceedings of the 21st ACM SIGKDD International Conference on Knowledge Discovery and Data Mining, pp. 1721-1730, ACM, Sydney, Australia, August 2015.

[30] F. Ming, C. Du, Y. Liu, X. Shi, and D. Li, "Concrete durability under different circumstances based on multi-factor effects," Sciences in Cold and Arid Regions, vol. 9, no. 4, pp. 384-391, 2018.

[31] ISO 15686-1, Buildings and Construction Assets-Service Life Planning-Part 1: General Principles and Framework, ISO, Geneva, Switzerland, 2011.

[32] I. Goodfellow, Y. Bengio, and A. Courville, Deep Learning, MIT Press, Cambridge, MA, USA, 2016.

[33] N. J. Guliyev and V. E. Ismailov, "A single hidden layer feedforward network with only one neuron in the hidden layer can approximate any univariate function," Neural Computation, vol. 28, no. 7, pp. 1289-1304, 2016.

[34] A. B. Owen and C. Prieur, "On Shapley value for measuring importance of dependent inputs," SIAM/ASA Journal on Uncertainty Quantification, vol. 5, no. 1, pp. 986-1002, 2017.

[35] I. E. Kumar, S. Venkatasubramanian, C. Scheidegger, and S. Friedler, "Problems with Shapley-value-based explanations as feature importance measures," 2020, https://arxiv.org/abs/ 2002.11097.

[36] JMA, "Annual changes of carbon dioxide concentration by locations," Japan Meteorological Agency, 2019, https://ds. data.jma.go.jp/ghg/kanshi/co2timeser/co2timeser.html.

[37] MLIT, "Guidelines for general traffic survey," MLIT, 2010, http://www.mlit.go.jp/road/census/h22-1/data/kasyorep.pdf.

[38] MLIT, "National road and street traffic situation survey (general traffic volume survey)," 2015, http://www.mlit.go.jp/ $\mathrm{road} /$ census/h27/index.html.

[39] R. Kobayashi, "Survey on salinity penetration of existing bridges along the coast of oshima peninsula, Hokkaido, special report on survey report at 2009 diagnosis workshop," Concrete Diagnosis Page, vol. 48, no. 7, pp. 52-55, 2010.

[40] JSCE, Standard Specifications for Concrete Structures (Design), Japan Society of Civil Engineers, JSCE Guidelines for Concrete, Tokyo, Japan, 2012.

[41] H. Yokota, "Practical application of life-cycle management system for shore protection facilities," Structure and Infrastructure Engineering, vol. 13, no. 1, pp. 34-43, 2017. 